\title{
Application of Evolutionary Algorithms and Statistical Analysis in the Numerical Optimization of an Axial Compressor
}

\author{
Alberto Clarich \\ Department of Energetics, Faculty of Engineering, University of Trieste, 10-34127 Trieste, Italy \\ Email: clarich@units.it

\section{Giovanni Mosetti} \\ Department of Energetics, Faculty of Engineering, University of Trieste, 10-34127 Trieste, Italy \\ Email:mosetti@univ.trieste.it \\ Valentino Pediroda \\ Department of Energetics, Faculty of Engineering, University of Trieste, 10-34127 Trieste, Italy \\ Email:pediroda@univ.trieste.it

\section{Carlo Poloni} \\ Department of Energetics, Faculty of Engineering, University of Trieste, 10-34127 Trieste, Italy \\ Email:poloni@univ.trieste.it
}

Received 25 June 2002

\begin{abstract}
The purpose of this work is to optimize the stator shape of an axial compressor, in order to maximize the global efficiency of the machine, fixing the rotor shape. We have used a 3D parametric mesh and the CFX-Tascflow code for the flow simulation. To find out the most important variables in this problem, we have run a preliminary series of designs, whose results have been analyzed by a statistic tool. This analysis has helped us to choose the most appropriate variables and their ranges in order to implement the optimization algorithm more efficiently and rapidly. For the simulation of the fluid flow through the machine, we have used a cluster of 12 processors.
\end{abstract}

Keywords and phrases: axial compressor, evolutionary optimization, statistical analysis.

\section{INTRODUCTION}

The axial compressor whose rotor shape we want to optimize is an experimental compressor built in 1992 in the Department of Energetics of Trieste University (Manzoni [8]). The hub of the machine is represented in Figure 1 with a detail of the rotor and stator blades, while, in Table 1, we describe the machine global performances relatively to the best efficiency point.

The rotor and stator blades are realized through the use of NACA $65-\left(\mathrm{cl}_{0}\right) 10$ profiles (NACA [11]), whose characteristics are defined for each blade section in Table 2. These profiles are the classical low subsonic compressors ones, char-

This is an open access article distributed under the Creative Commons Attribution License, which permits unrestricted use, distribution, and reproduction in any medium, provided the original work is properly cited. acterized by an arc of circle as camber and by a maximum thickness of $10 \%$ of the chord length.

The blade angle is defined by the chord and the machine rotation axis, while the lift coefficient of the isolated profile $\mathrm{cl}_{0}$ defines how much the camber ordinate is scaled relatively to the base profile NACA 65-(10)10, characterized by $\mathrm{cl}_{0}=1.0$. To conclude the definition of the rotor and of the stator, the number of blades is fixed to 16 for the former and 21 for the latter, while the aspect ratio (ratio of the blade height and the profile chord length) is 2.0 for the stator and 2.2 for the rotor. As the blade height is $170 \mathrm{~mm}$ (the hub radius is $130 \mathrm{~mm}$ and the shroud radius is $300 \mathrm{~mm}$ ), the rotor profile chord length is $77 \mathrm{~mm}$ while the stator one is $85 \mathrm{~mm}$, and they are both constant in the radial direction.

The aspect ratio value chosen is rather low, and this choice is justified by the fact that the compressor is a 


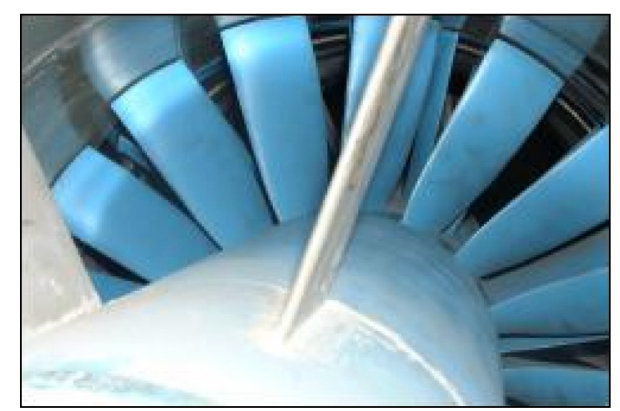

FIgURE 1: View of the axial compressor.

TABLE 1: Machine global performances.

\begin{tabular}{l|c}
\hline Rotation speed & $1210 \mathrm{rpm}$ \\
\hline Mass flow & $3.6 \mathrm{~kg}^{3} / \mathrm{s}$ \\
Total head & $300 \mathrm{~Pa}$ \\
Total efficiency & $85 \%$ \\
\hline
\end{tabular}

single-stage machine, and thus it requires a higher pressure load: a low aspect ratio usually gives higher load, higher efficiency, and higher stall pressure.

\section{PARAMETERIZATION AND NUMERICAL SIMULATION}

Since the optimization objective is the maximization of the machine efficiency, the first step of this job is the simulation of the flow through the compressor by a numerical fluiddynamic code.

We have defined a 3D multiblock-structured mesh, completely parametric, using the CFX-Tascflow commercial code (Figure 2).

The mesh is characterized by four main blocks. The two central ones contain the rotor and stator blade geometry, both defined properly by four sections.

As the machine is symmetric and the lateral surfaces of the two blocks are defined as periodic boundaries, the dimension of these blocks is influenced by the blade number of the rotor and of the stator. Beside them, there is also an inlet block, whose function is to drive the flow towards the rotor, and an outlet block, inside which the flow is free to expand as well as it would do in the external room.

For these reasons, we apply a boundary condition of mass flow fixed on the inlet $(3.6 \mathrm{~kg} / \mathrm{s}$ as defined by the project conditions) and a static pressure boundary on the outlet, fixed to the atmospheric value.

The numerical code applies the equation relative to the inertial body forces due to the rotation in the region defined by the rotor block, and thus it is necessary to define the interfaces between that block and the two contiguous ones as stage ones: with this option, CFX-Tascflow considers the numeric values of the variables in that region as medium ones, since the relative position of the two blocks is not frozen.
The numerical code is based on the Navier-Stokes equations, while the turbulence model used is the standard $k-\varepsilon$.

The total number of nodes in the mesh is about 300000 , and in particular, for the rotor and stator blocks, the nodes number in $i$-direction (inlet to outlet) is 100 , in $j$-direction (periodic to periodic) is 46 , while in $k$-direction (hub to shroud) is 40 .

The calculation convergence (maximum residuals under $4 e^{-4} \log$ ) is achieved after three hours using a double processor PENTIUM III $550 \mathrm{MHz} 128$ Mbytes, while as CFXTascflow allows the parallel computation, the calculation time has been considerably reduced (to about one hour) through a 5-processor line cluster, available on the CAD/CAE Laboratory of the Department of Energetics.

As we will see in the last chapter about the comparison of the flow fields, the flow seems quite irregular particularly in the stator, where the flow is detached from the blade and is characterized by the formation of vortex, thus it was decided to perform an optimization of the only stator, maintaining the rotor geometry unchanged, which was characterized in the simulation by a more regular flow field.

\subsection{Geometric parameterization}

In the stator shape parameterization, we have initially chosen 16 variables, which we describe in Table 3 .

From the initial geometry of the profile NACA 65-(10)10, defined by a standard camber and thickness, both dimensionless respects to the chord length, we have used these variables to change the blade geometry and to define four sections of the stator.

The variable ar, or aspect ratio, defines the ratio between the blade height, which is fixed to $170 \mathrm{~mm}$, and the chord length. The original stator was characterized by a value of ar of 2.0, and we have chosen a range of variation around that value.

With the chord length defined in that way, the camber of the NACA Profile is modified by adding to it, point to point, a third-order Bézier curve, which is defined by four control points.

The coordinates of these control points are defined by four variables, Bézier1-Bézier 4, which define the abscissa and ordinate, respectively, of the two central control points free to change their position.

The Bézier curve is a regular and continuous function, whose rates on the domain bounds are defined by the straight lines passing through the first two and the last two control points (Engeln-Mullges and Uhlig [3]).

The NACA profile thickness is also changed, multiplying the original value by the variable thick, which is made changing from 0.5 to 1.2 .

The new profile so-defined is rotated by different angles in the four sections placed on radii equal to $130,186,243$ and $300 \mathrm{~mm}$, respectively, and this angle is controlled by the variables psi 1-psi 4 .

While psi 1 , which varies from +5 to -5 degrees, shows how much the blade angle changes from the original value in 
TABLE 2: Features of the rotor and stator blade sections.

\begin{tabular}{l|cccc}
\hline Design parameters & Radius $(\mathrm{m})$ & $\mathrm{cl}_{0}$ & Blade angle (degrees) & NACA profile \\
\hline Rotor section 1 & 130 & 1.95 & 23.46 & $65-(20) 10$ \\
Rotor section 2 & 186 & 1.23 & 45.0 & $65-(12) 10$ \\
Rotor section 3 & 243 & 0.81 & 58.39 & $65-810$ \\
Rotor section 4 & 300 & 0.62 & 65.3 & $65-610$ \\
\hline Stator section 1 & 130 & 1.82 & 19.8 & $65-(18) 10$ \\
Stator section 2 & 186 & 2.12 & 21.8 & $65-(21) 10$ \\
Stator section 3 & 243 & 2.45 & 23.8 & $65-(25) 10$ \\
Stator section 4 & 300 & 2.82 & 24.8 & $65-(27) 10$ \\
\hline
\end{tabular}

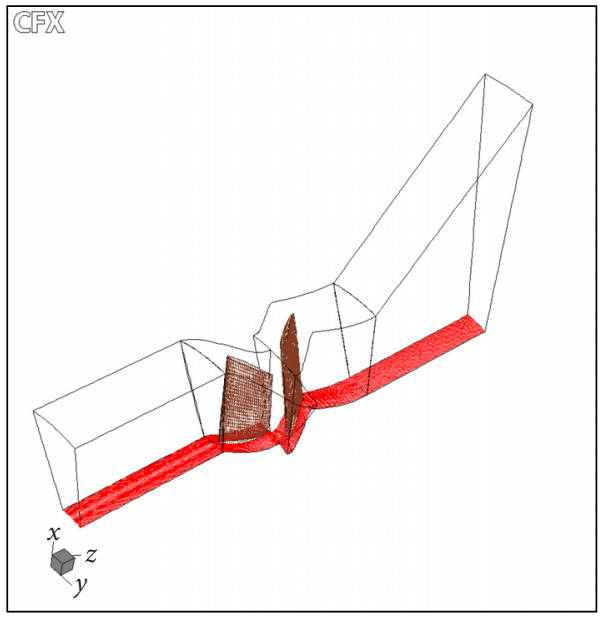

FIGURE 2: View of the mesh and of the calculation domain.

the section characterized by radius equal to $130 \mathrm{~mm}$, the variables psi 2-psi 4 show how much each one of the three angles is greater than the previous one, assuming that the blade angle grows in the radial direction.

The variables $\mathrm{cl} 1-\mathrm{cl} 4$ define instead how the curvature of the four profiles changes with respect to the original one. To obtain the camber of the first profile, we have to add to the original coefficient $\mathrm{cl}$ of the section $r=130 \mathrm{~mm}$ the value of the variable $\mathrm{cl} 1$, while the other three variables $\mathrm{cl} 2-\mathrm{cl} 4 \mathrm{de}-$ fine how much the coefficient $\mathrm{cl}$ changes with respect to the previous section, assuming also in this case that the camber grows in the radial direction.

Since the coefficient cl of a section is known, it is possible to multiply the camber ordinates by it, obtaining a higher or lower curvature.

Finally, the number of stator blades is defined by the variable nblade, which will influence the stator block dimension, while the variable stagg defines the value of the stagger in the tangential direction.

In other words, the barycentre of the stator sections is placed not on the same radial line, but on a line which is shifted by a certain angle in the tangential direction. The tangent of this angle is defined by the ratio between the variable stagg and the shroud radius $(300 \mathrm{~mm})$.
TABLE 3: Sixteen initial variables and their range of variation.

\begin{tabular}{l|cc}
\hline Blade number & nblade & $(16,23)$ \\
Blade thickness & thick & $(0.5,-1.2)$ \\
Stagger & stagg & $(-50,50)$ \\
Aspect ratio & ar & $(1.5,2.5)$ \\
Coefficient cl, section 1 & cl 1 & $(-0.4,0.4)$ \\
Blade angle, section 1 & psi 1 & $(-5,5)$ \\
Coefficient cl, section 2 & cl 2 & $(0.1,0.5)$ \\
Blade angle, section 2 & psi 2 & $(1,3)$ \\
Coefficient cl, section 3 & cl 3 & $(0.1,0.5)$ \\
Blade angle, section 3 & psi 3 & $(1,3)$ \\
Coefficient cl, section 4 & cl 4 & $(0.1,0.5)$ \\
Blade angle, section 4 & psi 4 & $(1,3)$ \\
Parameter Bézier 1 & Bézier 1 & $(0,1)$ \\
Parameter Bézier 2 & Bézier 2 & $(-0.25,0.25)$ \\
Parameter Bézier 3 & Bézier 3 & $(0,1)$ \\
Parameter Bézier 4 & Bézier 4 & $(-0.25,0.25)$ \\
\hline
\end{tabular}

\section{OPTIMIZATION WORKFLOW}

As stated before, the stator shape optimization has been performed by the using of statistical methodologies, as the high number of variables and the untrivial choice of their ranges of variation might have influenced negatively the searching of the best solutions.

The development of numerical techniques as well as $\mathrm{CAD} / \mathrm{CAE}$ methodologies has enforced the use of optimization algorithms, whose purpose is the minimization of the simulations number needed to obtain the optimal solution as soon as possible.

Several techniques have been set up to perceive this objective (Poloni et al. [6]; Poloni and Pediroda [7]; Catalano and Dadone [1]), among whom we have chosen to adopt the one based on the DOE (design of experiments) statistic methodology (Del Vecchio [2]).

Thus the optimization has been run in three phases: in the first two ones, 64 different individuals, or different configurations of the statoric blade, have been simulated, respectively, on the basis of a DOE methodology. By the use of this methodology it was possible to reduce the number of variables to 10 and to 6 , respectively, fixing the other variables 
that seemed to be less significative by this analysis. In the third phase, the gradient method was used to optimize locally an already good solution. The choice of the most significative variables has been conducted through the statistic analysis based on the Student parameter (Press et al. [9]), of which we report in the next paragraph a brief theoric description.

\subsection{Student parameter}

A DOE methodology consists of the selection of a certain number, that we indicate as $r$, of configurations, each one characterized by a different value assumed by each one of the $n$ variables of the problem.

Each variable may assume a value that may be represented by the symbol + or by the symbol -, depending on the fact that the value belongs to the upper half or to the lower half of its range.

The DOE set of configurations will then contain for each variable the same number, $r / 2$, of individuals characterized by the symbol + and by the symbol - corresponding to that variable.

In this way, it is possible to deal with a sufficiently large statistics in order to analyze properly the influence of each variable in the process, and it would be less probable to have data too localized for any variable.

As soon as we find the value of the fitness function for every configuration, it is possible to make a simple statistical analysis.

For each variable, in fact, we are able to calculate the mean value of the fitness function relative to the configurations characterized by the same symbol + assumed by that variable and the mean value of the same fitness function relative to the configurations characterized by the same symbol -. The difference of these means, divided by the deviation standard of the means, gives the Student parameter, which is a tool useful to estimate the percentage significance of the difference.

If this value is particularly high, it would be more probable that the two data populations are actually separated, it means that a high value of the variable (symbol + ) or a low value of the variable (symbol -) will produce a significative variation of the fitness function. If on the contrary, the Student parameter is low, it would be more probable that the data population is a single one, and thus the variable does not produce significative variation in the fitness function,

$$
\sigma=\sqrt{\frac{\left(\sum_{i=1}^{n_{1}}\left(x_{1 i}-\bar{x}_{1}\right)^{2}+\sum_{i=1}^{n_{2}}\left(x_{2 i}-\bar{x}_{2}\right)^{2}\right)\left(n_{1}+n_{2}\right)}{\left(n_{1}+n_{2}-2\right) n_{1} n_{2}}} .
$$

In (1), we report the function which defines the standard deviation of the mean values.

The two populations are characterized by $n_{1}$ and $n_{2}$ individuals defined by values + and - of the variable $x$, while the values of the fitness function are indicated by $x_{1 i}$ and $x_{2 i}$, respectively. We indicate the mean values of the fitness func-

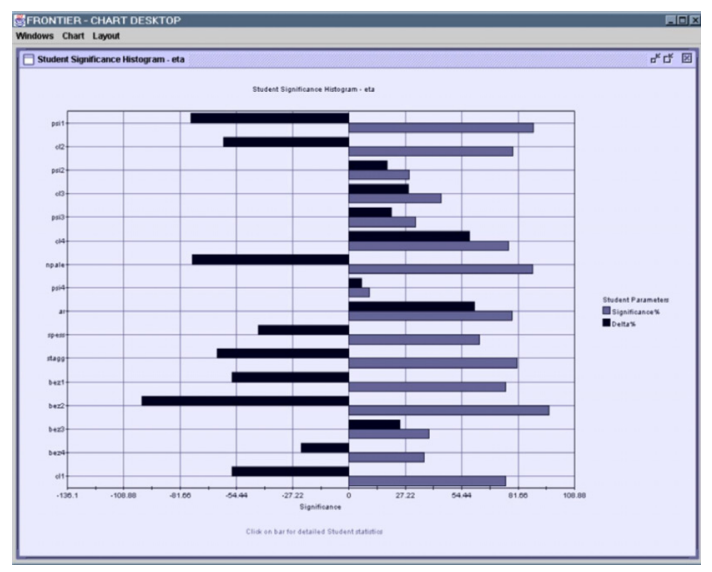

Figure 3: Significance of the variables in step 1.

tion for the two populations by $x_{1}$ and $x_{2}$ oversigned:

$$
t=\frac{\operatorname{abs}\left(\bar{x}_{1}-\bar{x}_{2}\right)}{\sigma} .
$$

In (2), we report instead the expression which defines the Student parameter. To each value of $t$, it is possible to associate, through a table which depends on the total number of individuals $n_{1}+n_{2}$, a significance percentage that expresses how much the variable is important for the fitness function, that means how much the latter is influenced by a variation of the variable.

In the next three paragraphs, we describe how we have performed the stator optimization, explaining which procedures have been employed and how the statistic methods have been integrated for the significance analysis of the variables.

\subsection{Step 1}

In the first step of the optimization, we have considered the 16 variables of Table 3, and a DOE table was produced of 64 different individuals, for which each variable assumed 32 times a value of the lower half of the range and 32 times a value of the higher half.

Each individual assumed a different value of the fitness function, which was defined by the total efficiency of the machine, calculated in function of the outflow power and of the power needed to balance the blades torque.

Among the different designs proposed in this first step, it was already possible to find some individuals characterized by a particular high value of efficiency, which was close to $93 \%$.

In Figure 3, we may note the value assumed by the Student parameter and the relative differences (delta) between the mean values of the individuals characterized by the symbols + and - for each variable.

We may note that as some variables, such as psi 4, are characterized by a very low value of significance percentage, we may decide to exclude them in the following phases of the 


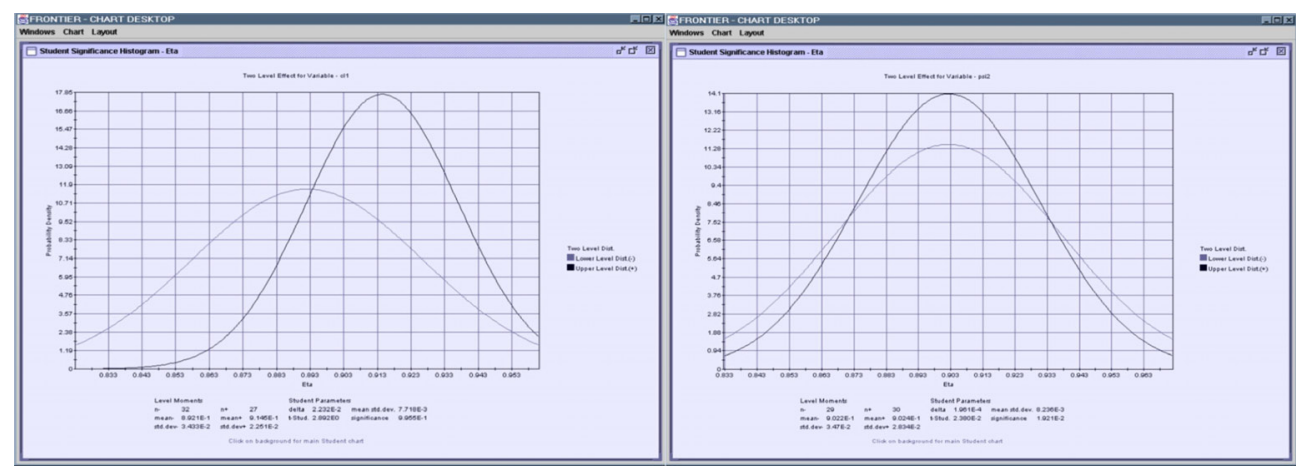

FIGURE 4: Comparison of populations characterized by individuals with high and low significances.

optimization, as they seem to have not much influence in the fitness function.

On the contrary, there are some variables, such as nblade and $\mathrm{cl}$, that seem to have a great influence in the process.

About the blade number, the higher value of the fitness function is achieved by the symbol - of the variable, that is, the value which belongs to the lower half of the range, while in the case of $\mathrm{cl} 1$, the higher value is achieved by the symbol + of the variable, that is, the one which belongs to the higher half of the range.

As an example, we may see in Figure 4 the comparison between a population of individuals characterized by a variable with a great influence in the fitness function and another one with a lower significance.

In abscissa, we report the values of the fitness function calculated for each individual of the population, while in ordinata, there are the relative frequencies. In each graphic, there are two Gaussian distributions, one relative to the individuals characterized by the symbol + and one relative to the individuals characterized by the symbol - referred to that variable. In the first case, the two Gaussian distributions are rather separated, it means that the difference of the two mean values relative to the standard deviation is high. In that case, the Student parameter is high, and we may deduce that the fitness function is highly influenced by that variable.

In the second case, instead, the two Gaussians are overlapped, and this gives a low value of the Student parameter. In fact, in this case, it becomes more probable that the two populations of individuals are not separated and that the fitness function seems to be less influenced by that variable, which we may not consider in a following phase of the optimization.

Repeating the same concept for all the variables, we may conclude that just 10 variables of the total 16 ones are more significative.

For this reason, in the second phase of the optimization, we have considered just these 10 variables, reducing or expanding their range of variation in the direction to which the fitness function seemed to grow, while the other 6 variables less significative have been fixed on the central values of their precedent ranges.
TABLE 4: Comparison of the variable ranges of the first and second optimization steps.

\begin{tabular}{l|cc}
\hline Design parameters & Step1 & Step2 \\
\hline nblade & $(16,23)$ & $(16,19)$ \\
thick & $(0.5,-1.2)$ & 1 \\
stagg & $(-50,50)$ & $(-50,0)$ \\
ar & $(1.5,2.5)$ & 2 \\
cl 1 & $(-0.4,0.4)$ & $(0,0.5)$ \\
psi 1 & $(-5,5)$ & $(0,5)$ \\
cl 2 & $(0.1,0.5)$ & 0.1 \\
psi 2 & $(1,3)$ & $(2,3)$ \\
cl 3 & $(0.1,0.5)$ & 0.1 \\
psi 3 & $(1,3)$ & 1 \\
cl 4 & $(0.1,0.5)$ & 0.1 \\
psi 4 & $(1,3)$ & $(2,3)$ \\
Bézier 1 & $(0,1)$ & $(0,0.5)$ \\
Bézier 2 & $(-0.25,0.25)$ & $(-0.25,0)$ \\
Bézier 3 & $(0,1)$ & $(0,0.5)$ \\
Bézier 4 & $(-0.25,0.25)$ & $(0,0.25)$ \\
\hline
\end{tabular}

In Table 4, we show the comparison between the ranges of the 16 variables of the first step and the ranges of the 10 variables of the second step.

In dark field, we represent the 6 variables that have been fixed in the second phase of the optimization, while in light field, we have the modified ranges of the other 10 variables more significative.

For example, the range relative to the blade number has been reduced in the direction of the lower half (from 16-23 to $16-19)$, as the symbol - referring to that variable gives a higher value of the fitness function, while the range of the variable $\mathrm{cl} 1$ has been reduced to the higher half for the opposite reason.

\subsection{Step 2}

In this second phase of optimization, there have been other 64 individuals simulated by applying another DOE model for 


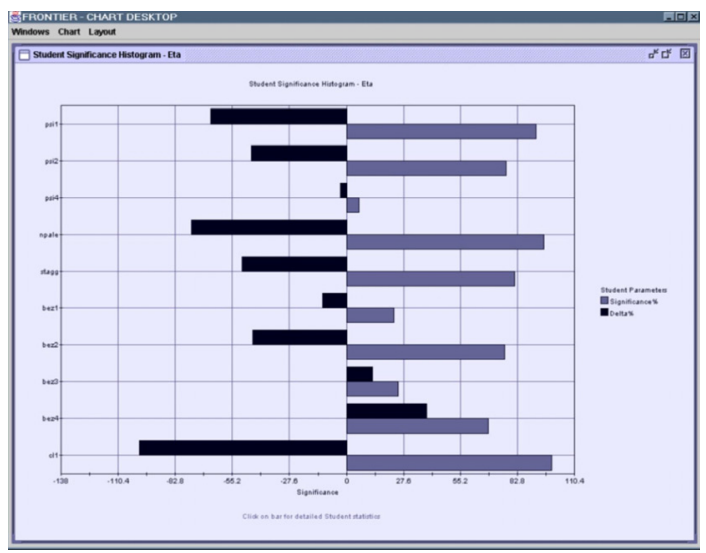

FIgURE 5: Variables significance in step 2.

TABle 5: Comparison between the variable ranges in the three phases of the optimization.

\begin{tabular}{l|ccc}
\hline Design parameters & Step1 & Step2 & Step3 \\
\hline nblade & $(16,23)$ & $(16,19)$ & 18 \\
thick & $(0.5,-1.2)$ & 1 & 1 \\
stagg & $(-50,50)$ & $(-50,0)$ & $(-60,-25)$ \\
ar & $(1.5,2.5)$ & 2 & 2 \\
cl 1 & $(-0.4,0.4)$ & $(0,0.5)$ & $(0,0.25)$ \\
psi 1 & $(-5,5)$ & $(0,5)$ & $(2.5,5)$ \\
cl 2 & $(0.1,0.5)$ & 0.1 & 0.1 \\
psi 2 & $(1,3)$ & $(2,3)$ & $(2,2.5)$ \\
cl 3 & $(0.1,0.5)$ & 0.1 & 0.1 \\
psi 3 & $(1,3)$ & 1 & 1 \\
cl 4 & $(0.1,0.5)$ & 0.1 & 0.1 \\
psi 4 & $(1,3)$ & $(2,3)$ & 2.5 \\
Bézier 1 & $(0,1)$ & $(0,0.5)$ & 0.25 \\
Bézier 2 & $(-0.25,0.25)$ & $(-0.25,0)$ & $(-0.35,-0.1)$ \\
Bézier 3 & $(0,1)$ & $(0,0.5)$ & 0.25 \\
Bézier 4 & $(-0.25,0.25)$ & $(0,0.25)$ & $(0,0.125)$ \\
\hline
\end{tabular}

the 10 variables considered. Also in this case, we report in Figure 5 the diagram relative to the Student parameter, which allows us to make other considerations about the significance of the variables.

In this case, we may note how just 6 variables are, relatively to their new ranges, still significative, so that we have decided to perform a last phase of optimization, applying an algorithm based on the gradient method, in order to optimize the fitness function relative to these 6 variables, choosing their range in function of the data obtained by the Student analysis.

In Table 5, we thus report the comparison between the ranges of the variables in the first two phases of the optimization and the ones of the 6 variables, in light field, remained in the last phase.

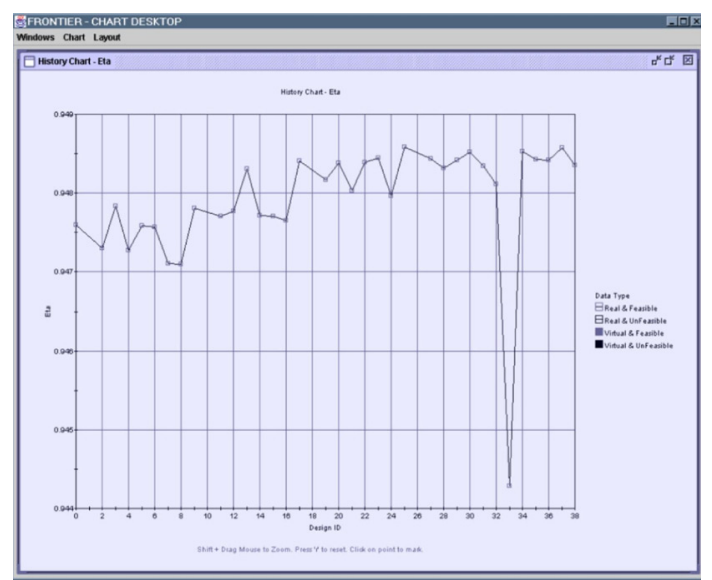

FIGURE 6: Course of the fitness function in the third optimization step.

\subsection{Step 3}

From the precedent Table 5, we may note how in the last phase of optimization we have considered just the variables stagg, cl 1, psi 1, psi 2, Bézier2, and Bézier4, while the other ones have been fixed on the central values of the ranges considered in the previous optimization step.

Through the gradient method and a low number of further simulations (38), it was possible to find a configuration characterized by an efficiency value equal to 0.948 , against 0.85 of the original configuration.

In Figure 6, we observe the course of the fitness function in this last optimization step.

\section{OPTIMIZED CONFIGURATION}

In Figure 7, we compare the original stator (thick line) with the optimized stator (thin line). We report the blade profiles relative to four different sections placed, respectively, to radii equal to $130,186,243$, and $300 \mathrm{~mm}$.

To understand how the efficiency has grown so much (from 0.85 to 0.948 ), we compare in Figure 8 the velocity field relative to three sections of the machine, one placed in proximity of the hub, one at middle height, and one close to the shroud, respectively, for the original and for the optimized configurations.

We may note that in the original configuration, the flow around the stator is characterized by a detachment, visible particularly in the lower side of the blade and partially in the higher one, while in the optimized configuration, it does not happen.

The stall in the original configuration is particularly due to the excessive curvature of the camber, which is much more reduced in the optimal configuration. In the latter one, in fact, while the angle of attack seems to be equal, the backside is much flatter and the outflow angle is not perfectly axial.

In this way, the flow is subjected to a weaker and more gradual deviation, avoiding the stall. 


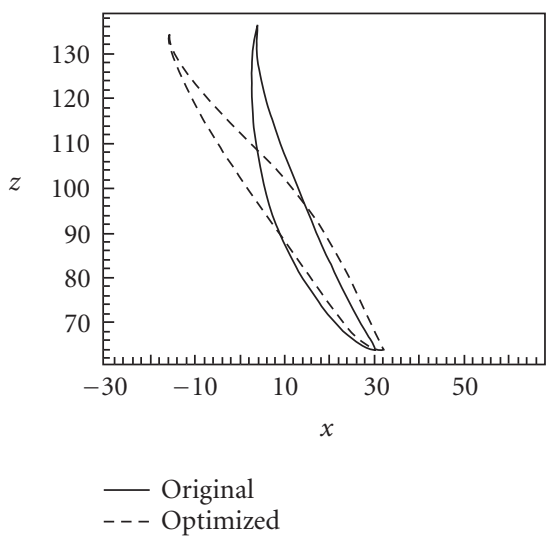

(a)

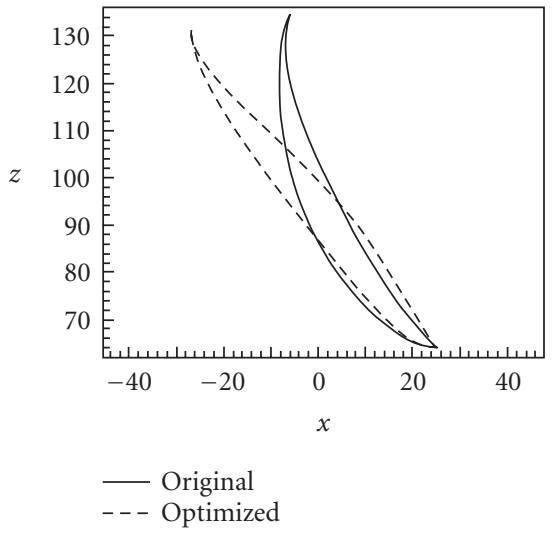

(c)

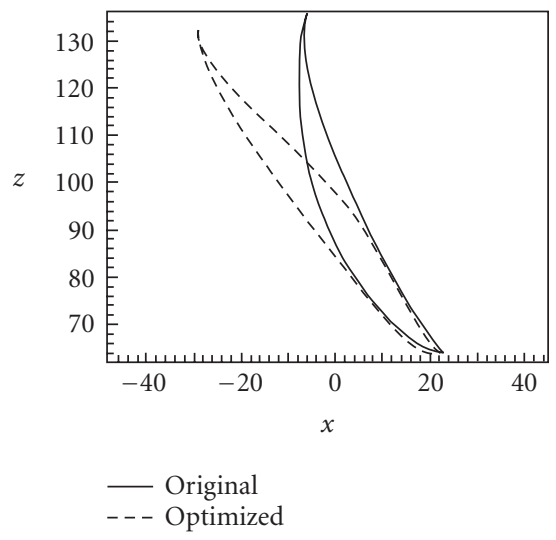

(b)

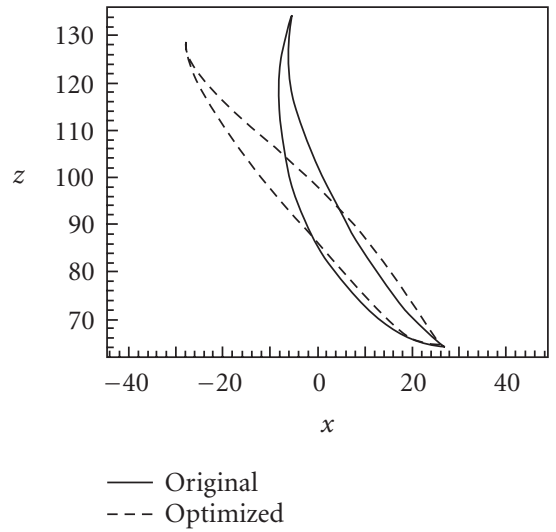

(d)

FIGURE 7: Comparison between the original stator blade profile and the optimized one: (a) section 1, (b) section 2, (c) section 3 , and (d) section 4 .

To confirm the important influence of the flow deviation on the efficiency, we may observe how the most significative parameters in the various phases of the optimization have been the ones concerning the profile camber, such as the Bézier parameters, the coefficient $\mathrm{cl}$ of the various section profiles, and the blade angle of the same ones (Table 5).

Among the other parameters, just the stagger has been significative, and the optimal solutions presented angles among 5 and 15 degrees.

We may thus conclude that this design methodology, based mainly on the statistic analysis of the problem, gives good results not only on the simulation (growth of the total efficiency of the machine), but it gives also a lot of information about the real importance of the parameters, supporting the designer in the choices and in the comprehension of the physics of the problem.

\section{CONCLUSIONS}

In this paper, we have described the shape optimization of an axial compressor stator in order to maximize the efficiency at the design point.
The parameterization of the stator shape $\mathrm{v}$ has required initially 16 variables, and, in order to reduce the analysis to the most significative ones, we have used a DOE methodology in two different steps.

In each phase, the statistical analysis has allowed us to find the variables that produced a most significative variation of the fitness function, so that it was possible to run a last phase of optimization, based on the gradient method, with the purpose of the maximization of the objective through a low number of individuals.

The application of the statistic method and of the evolutive algorithm has helped to improve the machine efficiency from 0.85 to 0.948 through the simulation of about 150 individuals, a number considerably low for the quantity of the problem variables.

\section{ACKNOWLEDGMENT}

We thank the ESTECO Ltd. for the use of Frontier code in the procedure of optimization. 


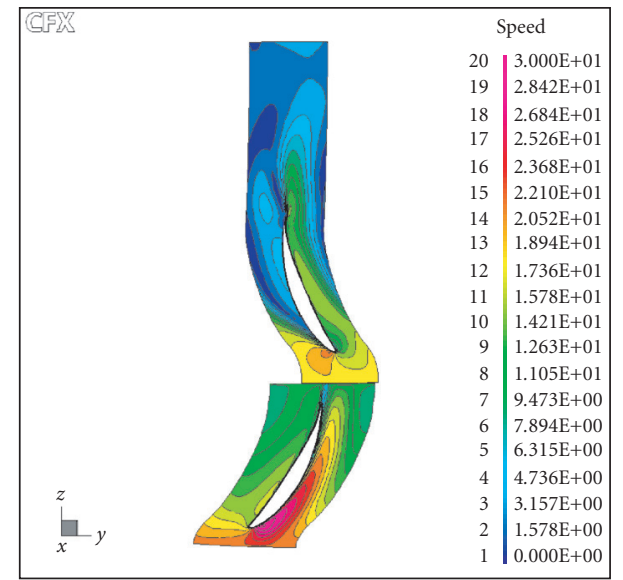

(a)

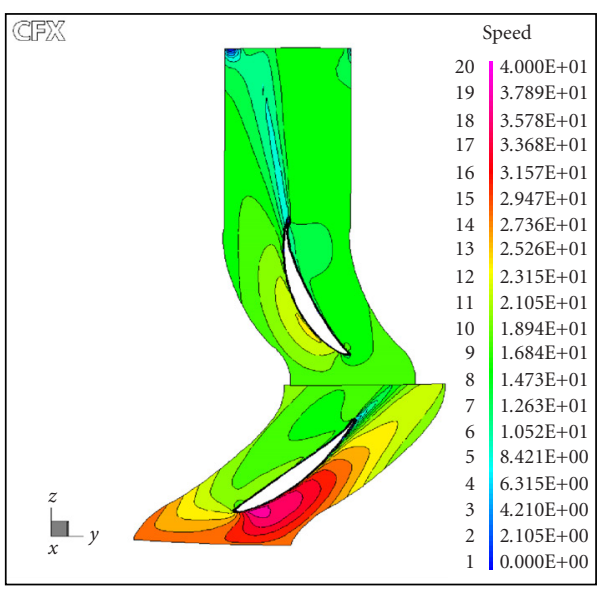

(c)

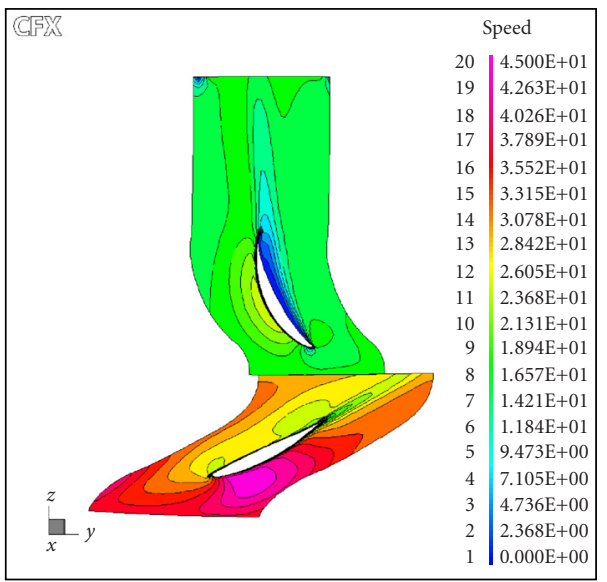

(e)

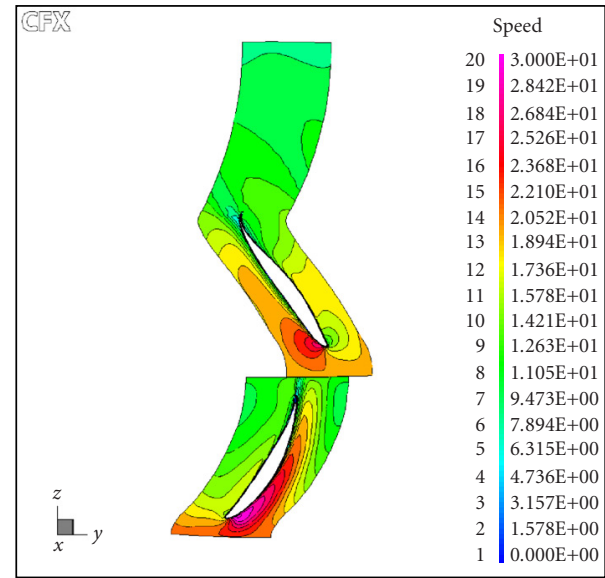

(b)

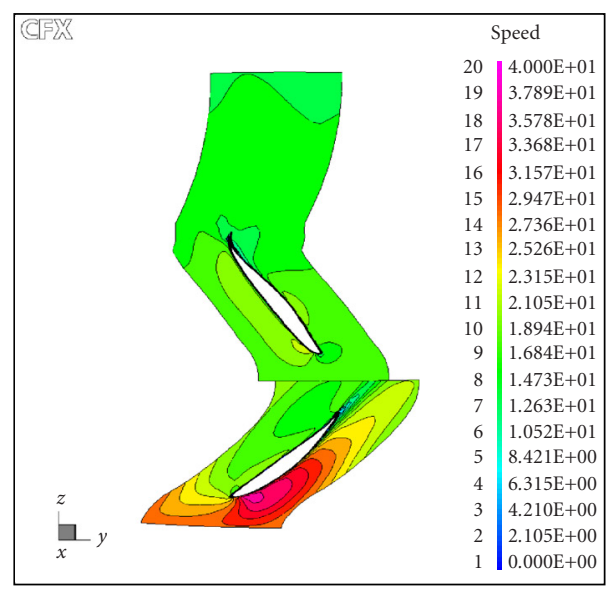

(d)

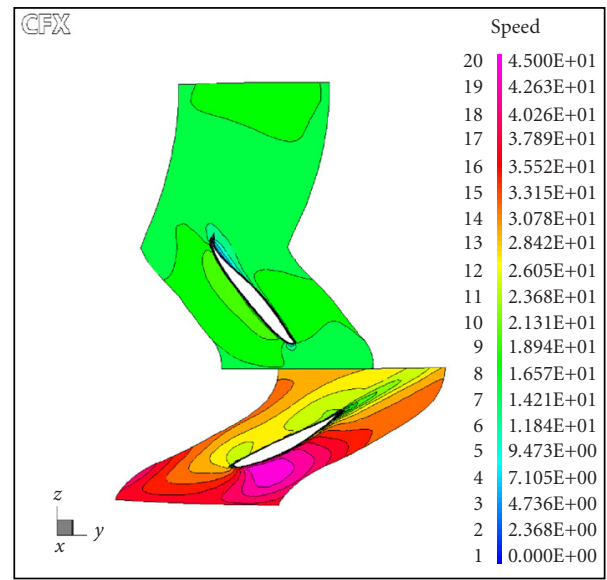

(f)

FIGURE 8: Comparison between the velocity field of the original configuration (left) and the optimized one (right), relatively to three sections, placed on 10,50 , and $90 \%$ of the blade height. 


\section{REFERENCES}

[1] L. A. Catalano and A. Dadone, "Progetto di schiere di pale transoniche mediante ottimizzazione progressiva," in Atti del Convegno $54^{\circ}$ Congresso Nazionale ATI, vol. 2, L'Aquila, Italy, September 1999.

[2] R. J. Del Vecchio, Understanding Design of Experiments, Hanser Understanding Books, Hanser Publishers, Munich, Germany, 1997.

[3] G. Engeln-Mullges and F. Uhlig, Numerical Algorithms with Fortran, Springer, Heidelberg, Germany, 1996.

[4] A. Garcia-Diaz and D. T. Phillips, Principles of Experimental Design and Analysis, Chapman \& Hall, London, UK, 1995.

[5] D. C. Montgomery, Design and Analysis of Experiments, John Wiley \& Sons, New York, NY, USA, 4th edition, 1997.

[6] C. Poloni, A. Giurgevich, L. Onesti, and V. Pediroda, "Hybridization of a multi-objective genetic algorithm, a neural network and a classical optimizer for a complex design problem in fluid dynamics," Computer Methods in Applied Mechanics and Engineering, vol. 186, no. 2-4, pp. 403-420, 2000.

[7] C. Poloni and V. Pediroda, "GA coupled with computationally expansive simulation: tool to improve efficiency," in $G e$ netic Algorithms and Evolution Strategies in Engineering and Computer Science, pp. 267-288, John Wiley \& Sons, Chichester, UK, 1998.

[8] G. Manzoni, Determinazione numerica delle caratteristiche fluidodinamiche, costruzione e verifica sperimentale della palettatura di un compressore assiale, tesi di laurea in Macchine speciali, Università degli Studi di Trieste, Trieste, Italy, 1992.

[9] W. H. Press, B. P. Flannery, S. A. Teukolsky, and W. T. Vetterling, Numerical Recipes Fortran Version, Cambridge University Press, Cambridge, UK, 1989.

[10] CFX-Tascflow-Version 2.8 User Documentation, 1998 AEA Technology Engineering Software Ltd.

[11] Tech. Rep. 1368, National Advisory Committee for Aeronautics (NACA), Washington, DC, USA, 1958. 

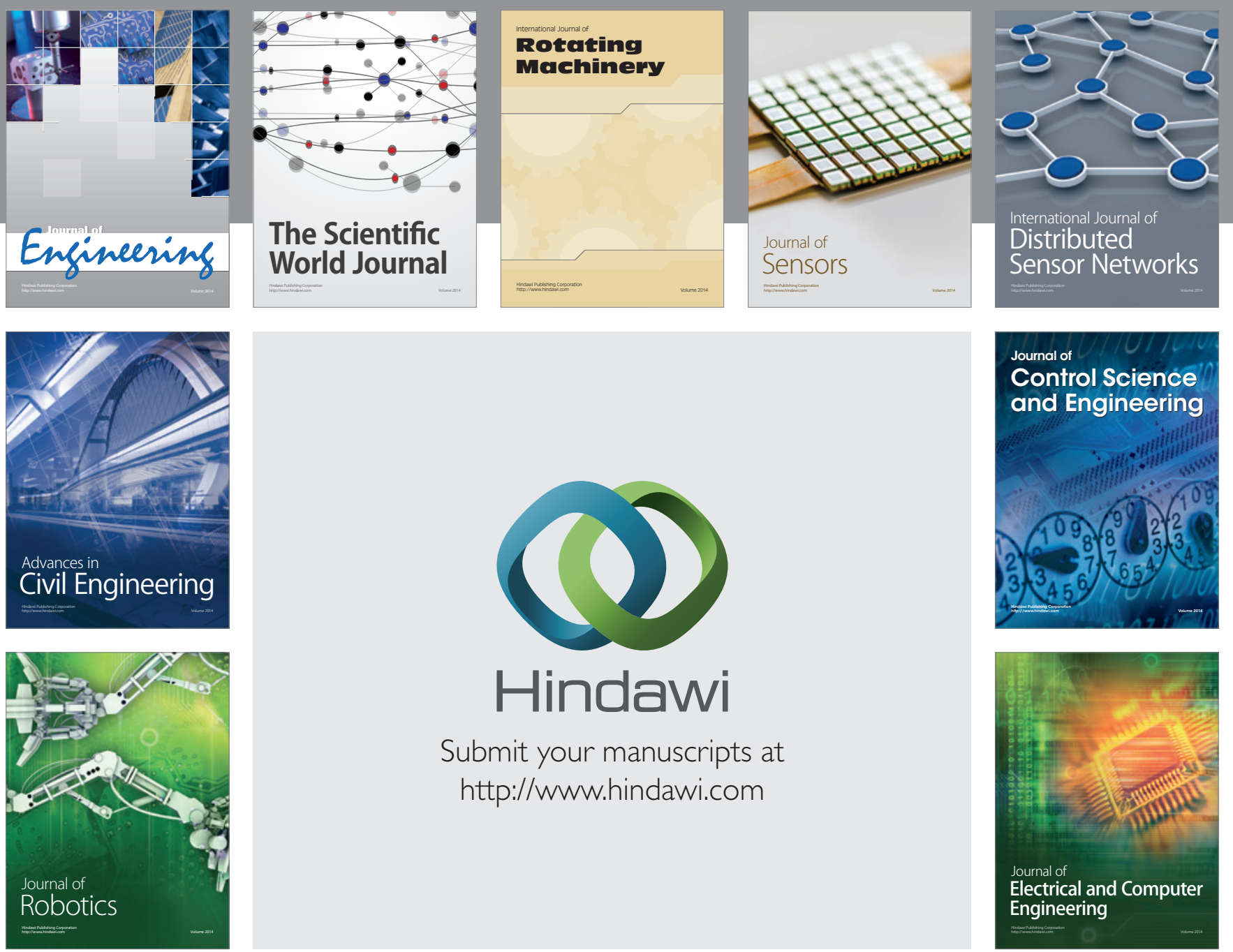

Submit your manuscripts at

http://www.hindawi.com
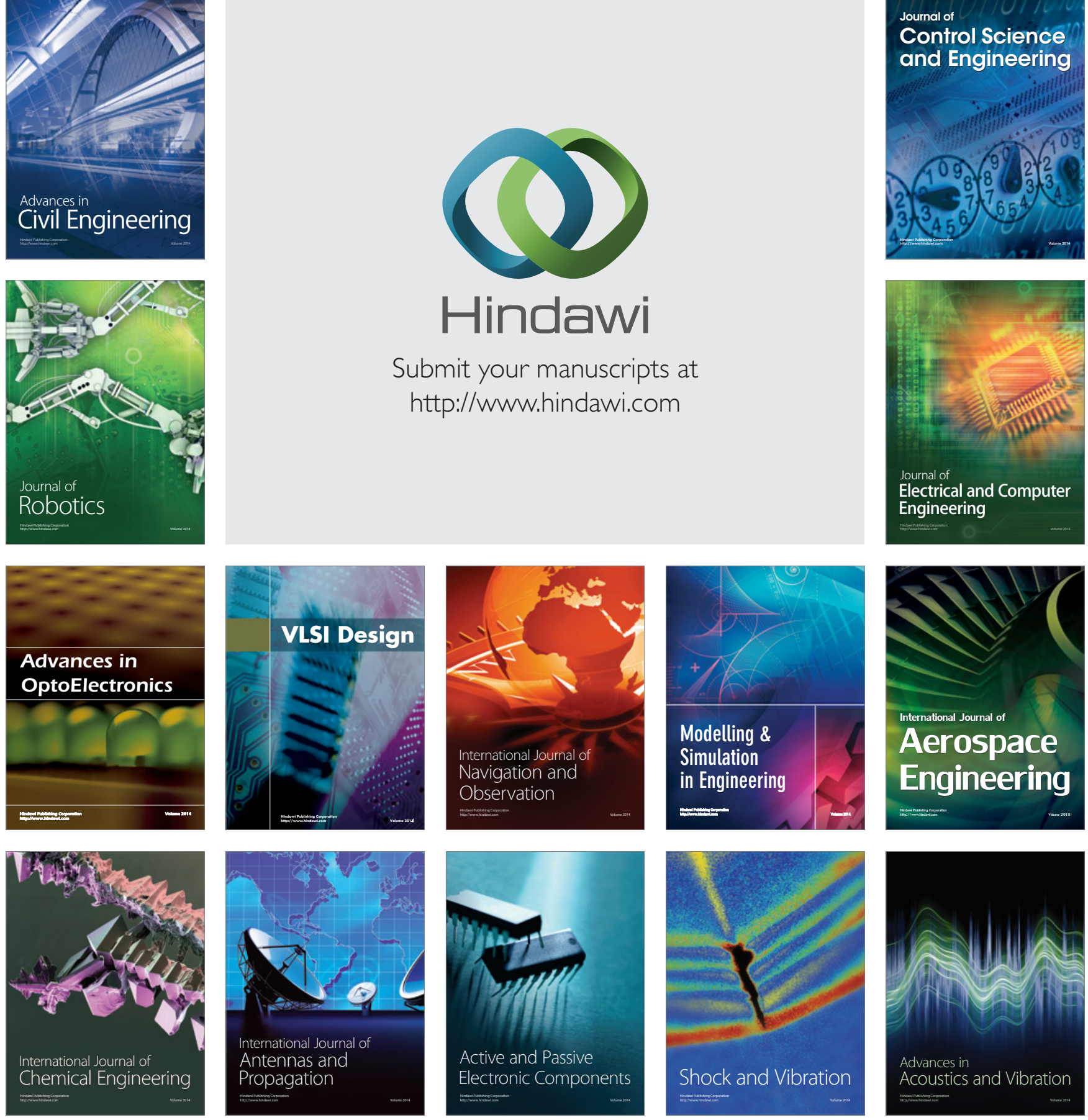